\title{
Fever of unknown origin as the first manifestation of Erdheim-Chester disease
}

\author{
Ning $\mathrm{Li}^{1^{*}}$, Mingquan $\mathrm{Chen}^{1^{*}}$, Huaping Sun ${ }^{2}$, Yun $\mathrm{Bao}^{3}$, Jiming Zhang ${ }^{1 \#}$ \\ ${ }^{1}$ Department of Infectious Diseases, Huashan Hospital, Fudan University, Shanghai, China; \\ ${ }^{\#}$ Corresponding Author: jmzhang@fudan.edu.cn \\ ${ }^{2}$ Department of Radiology, Huashan Hospital, Fudan University, Shanghai, China \\ ${ }^{3}$ Department of Pathology, Huashan Hospital, Fudan University, Shanghai, China
}

Received 8 July 2013; revised 1 August 2013; accepted 10 August 2013

Copyright (C) 2013 Ning Li et al. This is an open access article distributed under the Creative Commons Attribution License, which permits unrestricted use, distribution, and reproduction in any medium, provided the original work is properly cited.

\begin{abstract}
Erdheim-Chester disease (ECD) is an uncommon non-Langerhans cell histiocytosis that affects multiple body systems and can present clinically in a myriad of ways. We report the case of a 29-year-old man presented with fever and diffuse bone pain. Physical examination showed bilateral and symmetrical long bone pain, especially the knees. Radiographs showed multiple mixed bone lesions with sclerotic and lytic areas of the femora, tibiae, and fibula. Biopsy of the tibial area displayed foamy lipid-laden histiocytes, confirming the diagnosis of ECD.
\end{abstract}

Keywords: Erdheim-Chester Disease; Histiocytosis; Osteoarthropathy

\section{INTRODUCTION}

Erdheim-Chester disease (also known as ErdheimChester syndrome or polyostotic sclerosing histiocytosis, ECD) is a rare form of non-Langerhans-cell histiocytosis with particular tropism for connective and adipose tissues. To date, about 450 distinct cases of ECD have been described in the medical literature $[1,2]$. The number of new cases has dramatically increased over the past 10 years because of the better recognition of this condition.

ECD is a systemic disease characterized by proteiform manifestations, including skeletal involvement with bone pain, exophthalmos, diabetes insipidus, xanthelasma, interstitial lung disease, bilateral adrenal enlargement, retroperitoneal fibrosis with perirenal and/or ureteral obstruction, renal impairment, testis infiltration, central nervous system, and/or cardiovascular involvements. The pathology is characterized by foamy histiocytes or lipid-

\footnotetext{
*Ning Li and Mingquan Chen contributed equally to this work.
}

laden macrophages or histiocytes, surrounded by fibrosis, which are those with immunohistochemical findings demonstrating the presence of non-Langerhans histiocytes markers, namely, positivity for anti-CD68 labeling, little or no protein S100 labeling, and negativity for antiCD1a labeling $[3,4]$.

We present the case of a 29 -year-old male patient, who presented initially with decreased vision, diagnosis of VKHS. To the best of our knowledge, it is the first description of ECD with VKHS as the initial presentation. Leveled equations, graphics, and tables are not prescribed, although the various table text styles are provided. The formatter will need to create these components, incorporating the applicable criteria that follow.

\section{CASE REPORT}

A 29-year-old man was admitted to our hospital because of fevers, chills, cough, and pain in the both lower limbs. He presented a 10-month history of intermittent fevers (about $39^{\circ} \mathrm{C}$ ), progressive pain in both lower limbs, and nonproductive cough. There were numerous mildly enlarged bilateral cervical and supraclavicular lymph nodes. A doppler B scan system-guided fine-needle aspiration biopsy of the left cervical lymph nodes was performed, and pathological examination of the biopsy specimen revealed reactive lymphoid hyperplasia. A radiograph reportedly showed infection-related lesions in the tibia and fibula. Antibiotics sulperazon and vancomycin were administered without any improvement. Six days later, twice myeloid culture reportedly showed Human staphylococcus aureus, and bone scan reportedly showed lesions with increased activity involving the large joints. Antibiotics rifampicin, clindamycin, teicoplanin, or linezolid were sequentially administered, and the patient was running a mid to high fever, accompanied by lower limbs pain and discomfort. Antibiotics 
were discontinued, and antipyretic-analgesic was administrated. He had no prior medical history, and no exposure to occupational, dust, or animal hazards. He had no known allergies to medications. Medications included antipyretic-analgesic as needed for pain and fever, prednisone, potassium chloride sustained-release tablets, and pantoprazole. Laboratory examination results revealed erythrocyte sedimentation rate (ESR) of $90 \mathrm{~mm} / \mathrm{h}$ and Creactive protein (CRP) $96.5 \mathrm{mg} / 1$ (reference range, $<8$ ).

Approximately 8 weeks before admission, increased severe persistent pain in his right frontal, temporal, and occipital lobs were suffer from. Specimens of cerebrospinal fluid were stained for acid-fast bacilli, and cultured for bacteria, mycobacteria, and fungi and remained sterile. At the time of admission to our ward he appeared unwell, still fever, increasing fatigue, malaise, weakness, sweats, myalgias, and leg pain developed. The patient reported that the pain was located primarily in the region of both leg, occurred at rest, and awoke him at night. He described it as a constant aching and throbbing and gave it a score of 5 (on a scale of 1 to 10 , where 10 is the most severe pain). The pain lessened with rest and narcotic analgesia and increased when the patient walked and moved his leg.

His temperature was $39.5^{\circ} \mathrm{C}$, blood pressure $125 / 65$, pulse 106 per minute, respiratory rate 22 per minute. He had peripheral lymphadenopathy. B-ultrasound of the superficial lymph node showed multiple nodules throughout both bilateral cervical (up to $1.6 \mathrm{~cm}$ in diameter), supraclavicular lymph nodes (up to $2.1 \mathrm{~cm}$ in diameter), axillae (up to $1.4 \mathrm{~cm}$ in diameter) and the abdomen (up to $1.6 \mathrm{~cm}$ in diameter), and a thyroid nodule $(1.3 \mathrm{~cm})$. On abdominal examination, liver and spleen tip was not palpable. In our laboratory findings, results were normal for measurements of red and white blood cell count, platelets, glucose, plasma electrolytes, total protein, albumin, globulin, bilirubin, lactate dehydrogenase, immunoglobulins, and urine Bence Jones protein; studies of serum protein electrophoresis and tests of renal and liver function were also normal, as were serum levels of angiotensinconverting enzyme, antinuclear antibodies, rheumatoid factor, antineutrophil cytoplasmic antibodies (ANCA). Repeated culture of blood, sputum, urine, and stool were negative for possible pathogens.

To evaluate the extent of the disease, positron emission tomography/computed tomography (PET/CT) was performed $1 \mathrm{~h}$ after administration of $370 \mathrm{MBq} 18 \mathrm{~F}-$ fluorodeoxyglucose (FDG), which showed asystematic lytic bone lesion in the long bone and the pelvis with intensively FDG uptake (standardize uptake value, SUV approximately 15.5 to 17.1 ) (Figures 1(A)-(C)). There was mild FDG uptake (SUV, 2.4) with hyperdense nodules in bilateral posterior chamber of eyeball around optic nerves (Figure 1(D)). Malignant tumor was con-
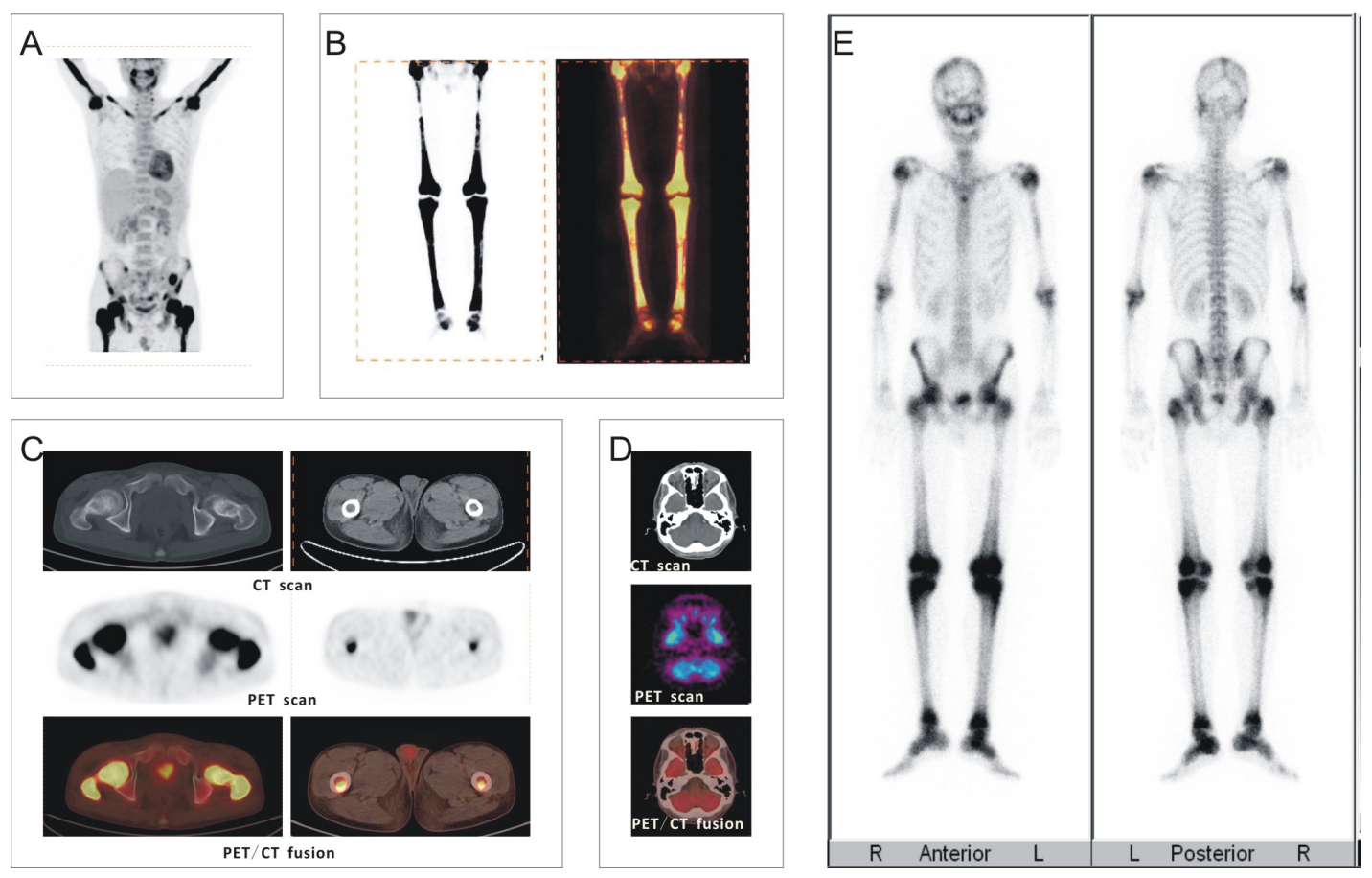

Figure 1. FDG PET/CT scan showed involvement of various organ systems in patients with Erdheim-Chester disease. (A)-(C) Typical bilateral and symmetric uptake in the diaphyseal and metaphyseal regions of the long bones was seen; (D) Neurologic involvement, with multiple nodules in bilateral posterior chamber of eyeball around optic nerves, is demonstrated; (E) A Tc-99m bone scintigraphy displayed a symmetrically increased uptake in the epiphyses of long bones, sparing the middiaphysis and the axial skeleton. 
sidered via PET/CT scan. However, anterior and posterior whole-body images of Tc- $99 \mathrm{~m}$ bone scintigraphy displayed the characteristic findings of ECD with symmetric increased radionuclide uptake in the epiphyses of long bones (Figure 1(E)). Findings of MRI showed replacement of the normal fatty bone marrow of the diaphyseal and metaphyseal bone segments by a markedly low signal intensity (hypointense compared with muscle and heterogeneous signal intensity on T1-weighted images) and heterogeneous intermediate or high signal intensity (isointense or hyperintense compared with muscle) on fatsuppressed T2-weighted images. Areas of high irregular signal intensity on $\mathrm{T} 1$-weighted images after contrast (Figure 2).

The patient had not been to rural areas or been exposed to animals recently. He reported no highrisk sexual activity, occupational exposures. Tests for histoplasma antigen were negative.
Five days later, at a follow-up visit, the patient reported increased leg pain. During the next 3 days, He was readmitted to this hospital. The vital signs were normal. There was mild percussion pain on both lower extremities, the remainder of the examination was unchanged. An electrocardiogram was normal. The whitecell count was 9200 per cubic millimeter, with a normal differential count. Specimens of blood and sputum were cultured for bacteria, mycobacteria, and fungi and remained sterile. Tests for respiratory viruses and antibodies to HIV were negative, as were multiple specimens of sputum stained for acid-fast bacilli. Two days later, an open excisional biopsy and curettage of the lesion of the left tibia were performed at this hospital; cefazolin was administered before and after the surgery; pathological examination of the biopsy specimen revealed infiltration of foamy histiocytes with irregular spindle cell proliferation and infiltration of mononuclear inflammatory cells
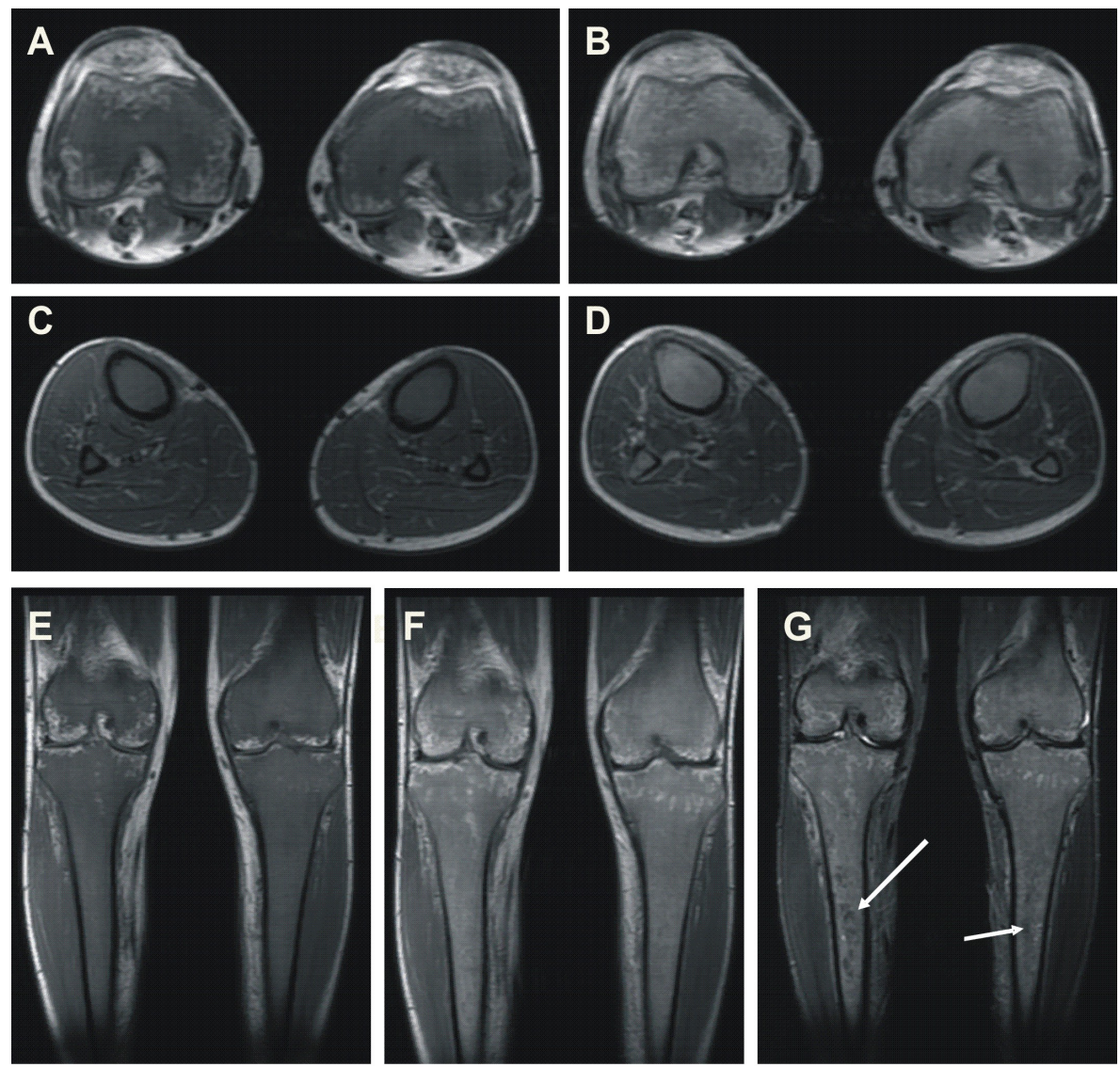

Figure 2. MRI findings. (A) Diffuse low signal intensity on axial T1-weighted images; and (B) intermediate enhancement on T1-weighted images after contrast are visible in diaphysis and metaphysis of distal femora; (C) Diffuse low signal intensity on axial T1-weighted images; and (D) with heterogeneous enhancement on T1-weighted images enhanced after gadolinium injection are visible in diaphysis and metaphysis of bilateral fibulas and tibias; (E) Diffuse low signal intensity on coronal T1-weighted images; and (F) irregular enhancement on T1weighted images after gadolinium injection are visible in diaphysis and metaphysis of distal femora and proximal tibiae; $(G)$ Fat-suppressed T1-weighted images after contrast show heterogeneous signal intensity, irregular stippled fat-suppressed signal (arrow). 
and eosinophils (Figure 3). Immunohistochemical staining showed infiltrating cells to be positive for CD68, lysozyme (LYS), but negative for S-100, CD1a. Based on the imaging and pathologic data, the diagnosis of ECD was made.template measures proportionately more than is customary. This measurement and others are deliberate, using specifications that anticipate your paper as one part of the entire journals, and not as an independent document. Please do not revise any of the current designations.

\section{DISCUSSION}

The first case of ECD was reported by W. Chester and Jakob Erdheim in 1930 [5]. ECD is a rare systemic nonLangerhans cell histiocytosis ( $\mathrm{LCH}$ ) with distinguishing clinicopathologic and radiographic features of unknown etiology. It is associated with many clinical presentations with involvement of 1 or more organ systems. In parti- cular, bone is affected, but skin, soft tissue, central nervous system, heart, lungs, or abdominal viscera can also be involved.

While the exact ontogeny is incompletely understood, histiocytes are believed to arise from a common CD-34 positive progenitor stem cell within the bone marrow. Two major types of histiocytes are described. Dendritic cells (e.g., Langerhans cells in skin and bronchial epithelium; and interdigitating and dendritic reticulum cells in spleen and lymph nodes) are the main antigen-presenting cells and are characterized by immunoreactivity to S-100 protein and CD1a [6]. The second main histologic cell type includes cells of the monocyte/macrophage lineage (e.g., circulating monocytes, alveolar macrophages, hepatic Kupffer cells, osteoclasts, and microglia) [7]. These antigen-phagocytosing cells are characterized by immunoreactivity to CD68. The causative cell of Langerhans cell histiocytosis $(\mathrm{LCH})$ is derived from the Langerhans
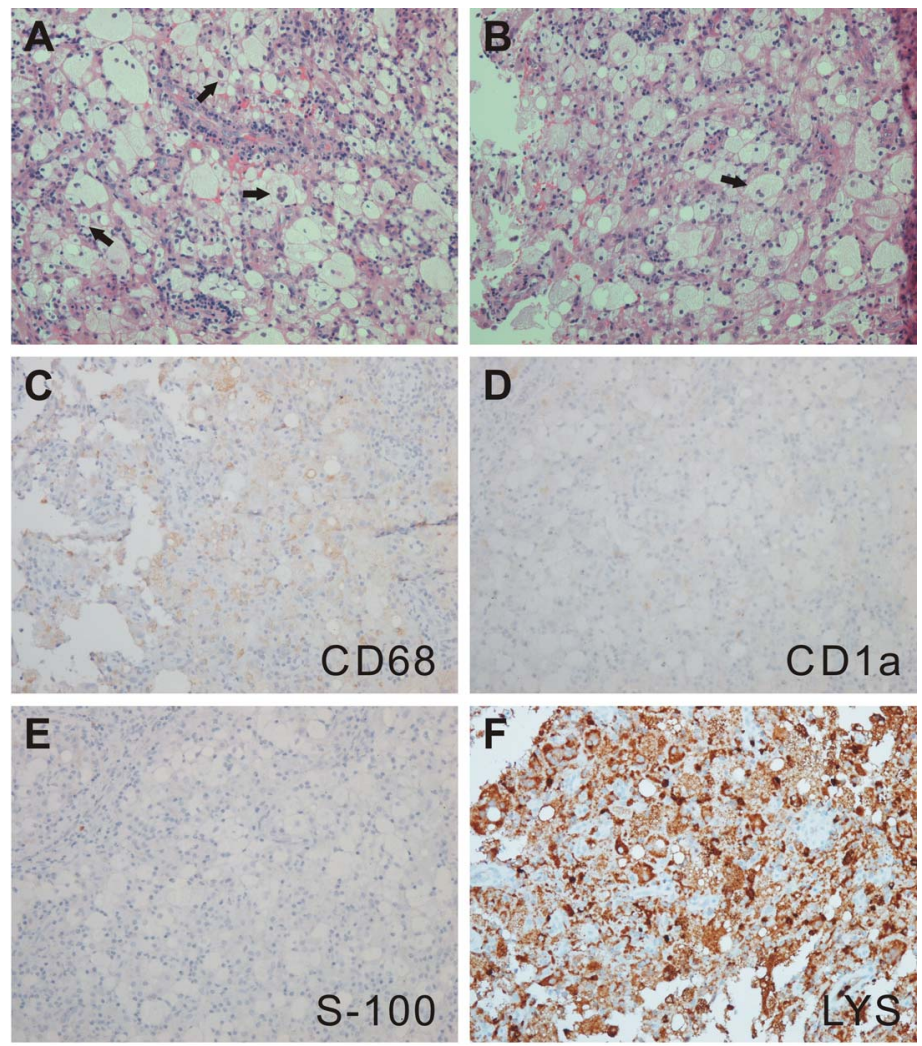

Figure 3. Histological photomicrographs. (A), (B) Intraoperative smear preparation showing foamy histiocytes (arrows) and small numbers of lymphocytes. Note presence of multinucleate giant cells (arrows) and emperipolesis. Paraffin-embedded, HE stain, $100 \times$ original magnification; (C) CD68 immunohistochemitry. Many of the foamy cells are immunoreactive. $100 \times$ original magnification; (D) CD1a immunohistochemistry. There is scarce immunoreactivity to CDla, a marker for certain dendritic cells. $100 \times$ original magnification; (E) S-100 immunohistochemistry. Only rare isolated cells are S-100 immunoreactive. 100× original magnification; (F) LYS immunohistochemistry. The cells are immunoreactive to LYS, $100 \times$ original magnification. 
dendritic cell line and that of ECD is from the monocyte-macrophage lineage, however, the clinical presentations can be similar, with bony involvement most frequent in both disease states. There are several key features, particularly immunohistochemical profile, that allow LCH and ECD to be distinguished.

The diagnosis of ECD is based on the combination of radiographic features, nuclear medicine features and nearly pathognomonic immunohistochemical profile as follow: evidence of bilateral and symmetrical cortical osteosclerosis of the diaphyseal and metaphyseal regions of the long bones and/or the symmetrical and strong uptake on the distal ends of the long bones of the legs and in some cases of the arms, on technetium-99 skeletal scintigraphy; and typical histological findings: xanthogranulomatous infiltration with foamy histiocytes surrounded by fibrosis; CD68-positive and CD1a-negative immunohistochemical labelling.

Patients with ECD typically develop characteristic multifocal osteosclerotic lesions of long bones [8] and may also develop extraskeletal histiocytic infiltrates [9]. Extra-osseous manifestations are numerous: central nervous system involvement may lead to diabetes insipidus [10], cerebellar symptoms, venous sinus thrombosis [11], and dural based masses, which may mimic multiple meningiomas $[12,13]$. Exopthalmos, which may be bilateral, occurs secondary to orbital infiltration. Pulmonary involvement is manifest as worsening dyspnoea, with interstitial infiltrates seen on chest radiographs [14]. Retroperitoneal fibrosis is a well-recognized sequela, and can cause bilateral adrenal infiltration [15], and also can lead to ureteric obstruction and progressive renal failure, which is a common cause of death in these patients.

When our case presented with only blurred and decreased vision involvement diagnosis of VKHS, after that persistent fever, increasing fatigue, malaise, weakness, and leg pain developed. The diagnosis of ECD was made until this case biopsy and curettage, according to the results of immunophenotypic analysis, which matches the ECD description. We think that immune factors may serve as an important stimulus for VKHS. Meanwhile, ECD lesions express a wide array of cyto-chemokines, which may orchestrate histiocyte recruitment and activation [16,17], and which is also related with immune factors. One of the unique points in our case is the progression from VKHS to ECD. The three most frequent clinical features of ECD are bone pain, diabetes insipidus and exophthalmos, these features are called the classic ECD triad. This patient only present with bone pain companied by fever, and twice myeloid culture reportedly showed Human staphylococcus aureus, which might have contributed to the delayed diagnosis; ECD is a very rare disease and difficult to diagnose, even with classic symptoms. However, the diagnosis of ECD can be made with near certainty because of the characteristic radiographic findings. The bilateral diffuse or patchy symmetric sclerosis of the medullary cavity of the major long bones, with relative epiphyseal sparing, is almost pathognomonic for ECD and can be confirmed by PET$\mathrm{CT}$. The distinct histological findings of ECD are also useful in making the diagnosis. Biopsy samples from the organs involved will reveal diffuse infiltrations of foamy histiocytes, Touton giant cells, lymphocytes and plasma cells, associated with dense fibrosis and reactive sclerosis of the cortical and cancellous bone. Immunohistochemistry of the biopsy samples confirms the monocyte/ macrophage lineage of the histiocytes by their expression of CD68, LYS, and S100 protein (variable), and by their lack of the Langerhans cell marker CD1a. This patient's biopsy material exhibited diffuse infiltration of CD68and LYS-expressing foamy histiocytes, and were negative for CD1a and S100. These findings were associated with dense fibrosis, which matches the ECD description. In this patient, additional immunohistochemical staining with antibodies against CD68 and CD1a supplemented the original histological findings. The present data, which are, to the best of our knowledge, it is the first description of ECD with VKHS as the initial presentation. It was difficult to assess the exact relationship between these 2 conditions.

ECD is a rare non-Langerhans histiocytosis of unknown etiology. The outcome of patients with ECD is worse than that for Langerhans-cell histiocytosis with $59 \%$ of patients in the former group dead after a mean follow-up of 32 months [3]. Although our study was not designed to assess the efficacy of treatments in ECD complicated by VKHS, these data suggest that corticosteroids which have been recognized as effective in other literature of VKHS, and have only a minor impact on the course of morbid involvement in ECD patients. Treatment strategies for ECD have been challenged by rare occurrence and poor understanding of etiopathology. Therapy with interferon- $\alpha$ (IFN- $\alpha$ ) effective in ECD appear to have minimal benefit [18-20]. A rationale for this approach is that IFN- $\alpha$ induces terminal differentiation of some histiocytes and dendritic cells. Targeting interleukin-1 (IL-1), a proinflammatory cytokine expressed by ECD histiocytes, with recombinant interleukin-1 receptor (IL-1R) has also recently been reported as a promising therapeutic strategy [1]. Arnaud and colleagues quantitatively analyze 23 cytokines in serum samples from 37 patients with ECD, and studied the impact of treatment on cytokine production [21]. They found no significant difference between cytokine levels measured in untreated and IFN- $\alpha$-treated patients. This suggests that IFN- $\alpha$ has limited impact on the systemic immunologic perturbations that are characteristic of ECD. They also found many significant differences in cyto- 
kine/chemokine levels in ECD patients compared with controls, with principal component analysis identifying an ECD-specific signature based on increased expression of IFN- $\alpha$, IL-1/IL-1RA, IL-6, IL-12, monocyte chemotactic protein-1, and decreased expression of IL-4 and IL-7. The authors conclude that the systemic immune Th-1-oriented perturbation associated with this condition, and provides clues for the choice of more focused therapeutic agents.

\section{CONCLUSION}

ECD is a rare form of non-Langerhans' cell histiocytosis. ECD appears to be the pathognomonic clinical, radiographic and histological features. Diagnosis of ECD is based on the identification in tissue biopsy of histiocytes, which are typically foamy and immunostain for $\mathrm{CD} 68+\mathrm{CD} 1 \mathrm{a}-$. Central nervous system involvement is a major prognostic factor in ECD. Because the incidence of ECD is low, the data from literature remains limited. More case data for this histiocytosis need therefore be obtained for a better understanding of its characteristics and an exploration of appropriate therapy.

\section{REFERENCES}

[1] Aouba, A., Georgin-Lavialle, S., Pagnoux, C., Silva, N.M., Renand, A., Galateau-Salle, F., et al. (2010) Rationale and efficacy of interleukin-1 targeting in ErdheimChester disease. Blood, 116, 4070-4076. doi:10.1182/blood-2010-04-279240

[2] Haroche, J., Arnaud, L. and Amoura, Z. (2012) ErdheimChester disease. Current Opinion in Rheumatology, 24, 53-59. doi:10.1097/BOR.0b013e32834d861d

[3] Veyssier Belot, C., Cacoub, P., Caparros Lefebvre, D., Wechsler, J., Brun, B., Remy, M., et al. (1996) ErdheimChester disease-Clinical and radiologic characteristics of 59 cases. Medicine, 75, 157-169. doi:10.1097/00005792-199605000-00005

[4] Wimpissinger, T.F., Schernthaner, G., Feichtinger, H. and Stackl, W. (2005) Compression of kidneys in ErdheimChester disease of retroperitoneum: Open surgical approach. Urology, 65, E29-E31. doi:10.1016/j.urology.2004.10.051

[5] Chester, W. (1930) The lipogranulomatosis. Virchows Archiv für Pathologische Anatomie und Physiologie und für Klinische Medizin, 279, 561-602. doi:10.1007/BF01942684

[6] Kairouz, S., Hashash, J., Kabbara, W., McHayleh, W. and Tabbara, I.A. (2007) Dendritic cell neoplasms: An overview. American Journal of Hematology, 82, 924-928. doi:10.1002/ajh.20857

[7] Zelger, B.W.H., Sidoroff, A., Orchard, G. and Cerio, R. (1996) Non-Langerhans cell histiocytoses-A new unifying concept. American Journal of Dermatopathology, 18, 490-504. doi:10.1097/00000372-199610000-00008

[8] Dion, E., Graef, C., Miquel, A., Haroche, J., Wechsler, B.,
Amoura, Z., et al. (2006) Bone involvement in ErdheirnChester disease: Imaging findings including periostitis and partial epiphyseal involvement. Radiology, 238, 632639. doi:10.1148/radiol.2382041525

[9] Allen, C.E. and McClain, K.L. (2011) Erdheim-Chester: Beyond the lesion. Blood, 117, 2745-2746. doi:10.1182/blood-2011-01-330233

[10] Wright, R.A., Hermann, R.C. and Parisi, J.E. (1999) Neurological manifestations of Erdheim-Chester disease. Journal of Neurology, Neurosurgery \& Psychiatry, 66, 72-75. doi:10.1136/jnnp.66.1.72

[11] Perras, B., Petersen, D., Lorch, H. and Fehm, H.L. (2002) Psychoneuroendocrine disturbances in a patient with a rare granulomatous disease. Experimental and Clinical Endocrinology \& Diabetes, 110, 248-252. doi:10.1055/s-2002-33075

[12] Naqi, R., Azeemuddin, M., Idrees, R. and Wasay, M. (2010) Meningioma-like lesions in Erdheim Chester disease. Acta Neurochirurgica, 152, 1619-1621. doi:10.1007/s00701-010-0655-0

[13] Donaldson, G., Bullock, P. and Monson, J.P. (2010) Erdheim-Chester disease mimicking multiple meningiomas. British Journal of Neurosurgery, 24, 296-297. doi: $10.3109 / 02688691003624612$

[14] Arnaud, L., Pierre, I., Beigelman-Aubry, C., Capron, F., Brun, A.L., Rigolet, A., et al. (2010) Pulmonary involvement in Erdheim-Chester disease: A single-center study of thirty-four patients and a review of the literature. Arthritis \& Rheumatism, 62, 3504-3512. doi:10.1002/art.27672

[15] Haroche, J., Amoura, Z., Touraine, P., Seilhean, D., Graef, C., Birmele, B., et al. (2007) Bilateral adrenal infiltration in Erdheim-Chester disease. Report of seven cases and literature review. Journal of Clinical Endocrinology \& Metabolism, 92, 2007-2012. doi:10.1210/jc.2006-2018

[16] Stoppacciaro, A., Ferrarini, M., Salmaggi, C., Colarossi, C., Praderio, L., Tresoldi, M., et al. (2006) Immunohistochemical evidence of a cytokine and chemokine network in three patients with Erdheim-Chester diseaseImplications for pathogenesis. Arthritis and Rheumatism, 54, 4018-4022. doi:10.1002/art.22280

[17] Dagna, L., Girlanda, S., Langheim, S., et al. (2010) Erdheim-Chester disease: Report on a case and new insights on its immunopathogenesis. Rheumatology, 49, 12031206. doi:10.1093/rheumatology/kep461

[18] Braiteh, F., Boxrud, C., Esmaeli, B. and Kurzrock, R. (2005) Successful treatment of Erdheim-Chester disease, a non-Langerhans-cell histiocytosis, with interferon-alpha. Blood, 106, 2992-2994. doi:10.1182/blood-2005-06-2238

[19] Jeon, I.-S., Lee, S.S. and Lee, M.K. (2010) Chemotherapy and Interferon-alpha treatment of Erdheim-Chester disease. Pediatric Blood \& Cancer, 55, 745-747. doi:10.1002/pbc.22636

[20] Suzuki, H.I., Hosoya, N., Miyagawa, K., Ota, S., Nakashima, H., Makita, N., et al. (2010) Erdheim-Chester disease: Multisystem involvement and management with interferon-alpha. Leukemia Research, 34, E21-E24. doi:10.1016/j.leukres.2009.07.026 
[21] Arnaud, L., Gorochov, G., Charlotte, F., Lvovschi, V., Parizot, C., Larsen, M., et al. (2011) Systemic perturbation of cytokine and chemokine networks in Erdheim-
Chester disease: A single-center series of 37 patients. Blood, 117, 2783-2790.

doi:10.1182/blood-2010-10-313510 